\title{
EDITORIAL
}

\section{Long and short of nerve conduction measures: reproducibility for sequential assessments}

With steady improvement and standardisation of methods, ${ }^{1}$ nerve conduction studies have become a reliable means of testing peripheral nerve function. They supplement clinical observation by precisely localising the lesion and characterising the conduction abnormalities. ${ }^{2}$ Delineating the extent and distribution of the neural lesion by this means also helps to quantify the degree of involvement. Optimal results can be expected only with proper choice of techniques, which in turn depends on the type of lesions under consideration.
Thus, the studies must be conducted as an extension of the clinical examination, rather than a laboratory test.

To document sequential changes for clinical follow up in general and for drug trials in particular, conduction studies must yield high sensitivity and reproducibility. A question often posed, but rarely tested in regard to these criteria relates to the length of the nerve segment under study. Other factors being equal, should shorter or longer segments be studied for better results?

A
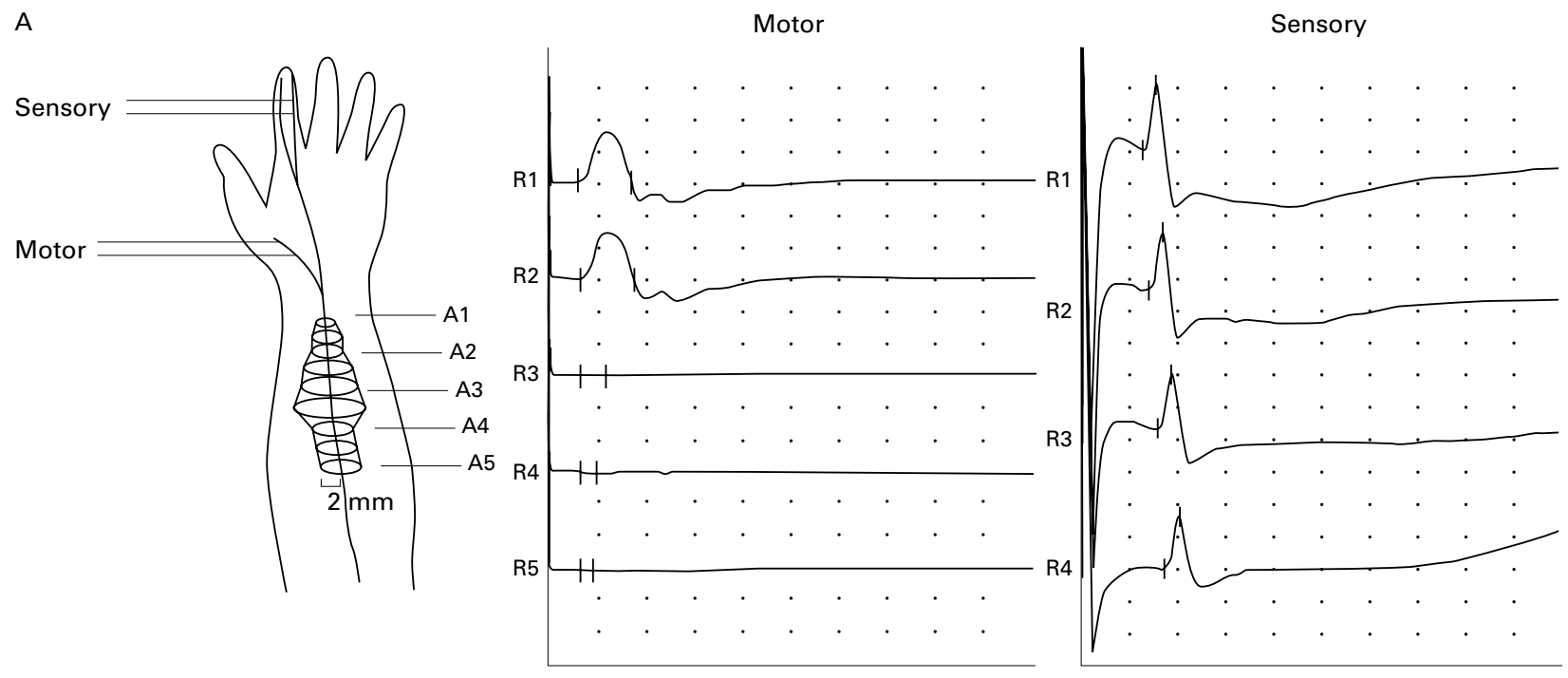

B

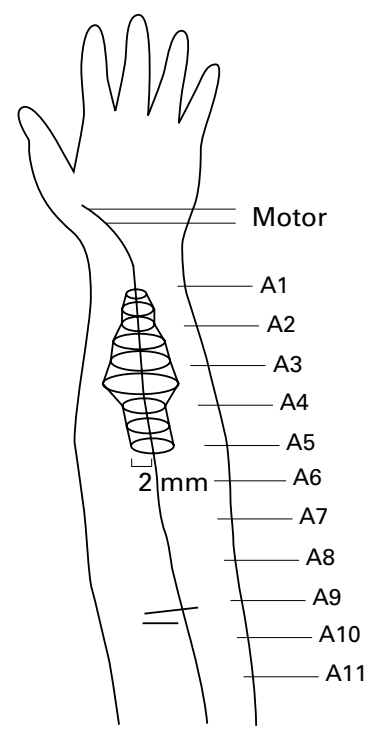

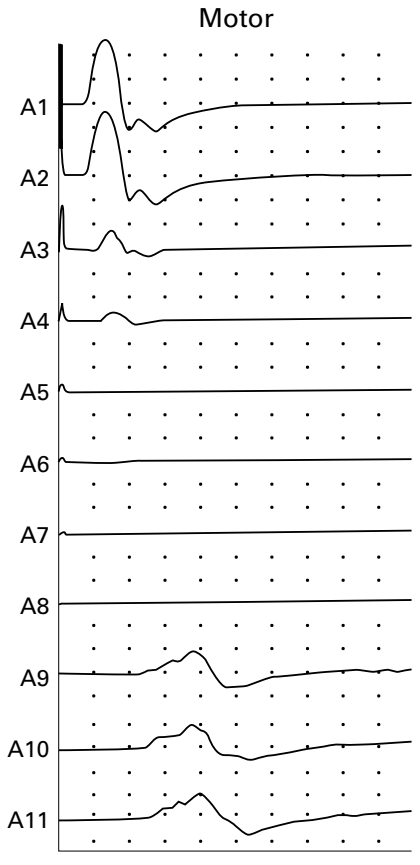

Figure 1 (A) Motor and sensory conduction studies of the left median nerve in a patient with multifocal motor neuropathy (top). The left diagram illustrates the consecutive slices of MR images in relation to the sites of stimulation at the wrist crease (A1) and at $2 \mathrm{~cm}$ increments more proximally. One horizontal division $=5 \mathrm{~ms}$ (motor) and $2 \mathrm{~ms}$ (sensory), and one vertical division $=5 \mathrm{mV}$ (motor) and $20 \mu \mathrm{V}$ (sensory) in $(A)$ and $2 \mathrm{mV}$ (motor) in (B). Note the complete and selective motor conduction block across the segment between $A 2$ and $A 3$, corresponding to the site of maximal nerve enlargement. Stimulation of the nerve at the elbow (not shown) also failed to elicit any motor response, confirming the block of impulse propagation rather than nerve inexcitability at the lesion site (from Kaji et al ${ }^{10}$ with permission). (B) A repeat study in the same patient after return of strength of the median innervated intrinsic hand muscles. High intensity stimulation failed to excite the nerve along the affected segments, $A 5-A 8$, mimicking a conduction block. More proximal stimulation at the elbow applied to the presumably normal nerve segments, A9-A11, however, induced a series of temporally dispersed muscle response associated with thumb abduction, indicating recovery of conduction (from Kimura ${ }^{25}$ with permission). 


\section{Segmental stimulation in short increments}

In the evaluation of a focal lesion, studies of a longer segment tend to lower the sensitivity of the test because the inclusion of the unaffected segments in calculation dilutes the effect of slowing at the site of lesion. By contrast, studying a shorter segment helps to isolate a localised abnormality and provides better resolution of restricted lesions that may otherwise escape detection.

For example, patients with carpal tunnel syndrome show a sharply localised latency increase, averaging $0.8 \mathrm{~ms}$ across a $1 \mathrm{~cm}$ segment. This, compared to a normal value ranging from 0.16 to $0.21 \mathrm{~ms}$, clearly indicates a focal abnormality. ${ }^{3}$ An abrupt change in waveform of the recorded response provides an additional, and perhaps more convincing, finding that nearly always accompanies an increase in latency across the site of compression. In fact, waveform analysis often localises a focal lesion unequivocally even in the absence of an abnormal latency prolongation.

This technique suits not only in assessing a possible compressive lesion such as carpal tunnel syndrome at the wrist, ${ }^{3-6}$ ulnar neuropathy at the elbow, ${ }^{78}$ and peroneal nerve entrapment at the knee, ${ }^{9}$ but also in characterising the focal nature of some widespread abnormalities such as multifocal motor neuropathies (fig 1 ). ${ }^{10}$

\section{Late responses for evaluation of long pathways}

Despite the traditional use of conduction studies across a relatively short distal portion of the peripheral nerves, a longer segment may provide a better result in assessing a more diffuse or multisegmental process such as polyneuropathies. A longer path has an advantage in accumulating all the segmental abnormalities, which individually might not show a clear deviation from the normal range. Thus, in general, the longer the segment under study, the more evident the conduction delay for a diffuse process. ${ }^{11}$

Assume a nerve impulse conducting at a rate of 0.2 $\mathrm{ms} / \mathrm{cm}(50 \mathrm{~m} / \mathrm{s})$; a $20 \%$ delay for a $10 \mathrm{~cm}$ segment is only $0.4 \mathrm{~ms}$, whereas the same change for a $100 \mathrm{~cm}$ segment amounts to $4.0 \mathrm{~ms}$, an obvious increase for easy detection. Evaluating a longer, as compared with a shorter, segment

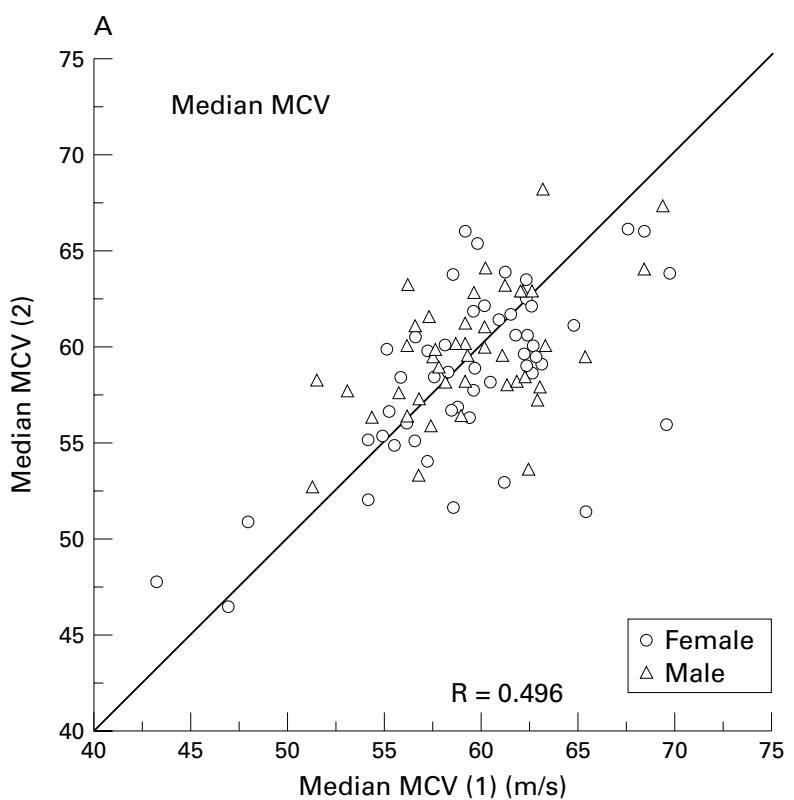

also improves the overall accuracy because the same absolute error leads to a smaller percentage of change in measuring either the latency or the distance. In routine practice, a surface measurement of a $10 \mathrm{~cm}$ nerve segment may yield an estimated distance of 9.5 to $10.5 \mathrm{~cm}$. A $1 \mathrm{~cm}$ difference constitutes a $10 \%$ error, or a calculated conduction velocity between $50 \mathrm{~m} / \mathrm{s}$ and $55 \mathrm{~m} / \mathrm{s}$. The same $1 \mathrm{~cm}$ error in a $100 \mathrm{~cm}$ segment represents only $1 \%$ error, or a conduction velocity between $50 \mathrm{~m} / \mathrm{s}$ and $50.5 \mathrm{~m} / \mathrm{s}$. The same argument holds in determining the effect of possible error in latency measurement. Thus, studying a longer path offers a better sensitivity and accuracy and, as stated later, improved reproducibility in serial studies.

Several neurophysiological methods supplement the conventional techniques for the assessments of longer pathways. ${ }^{12}$ The selection of such techniques necessarily reflects the special orientation of each laboratory. Those of general interest include the $\mathrm{F}$ wave and the $\mathrm{H}$ reflex.

\section{Reproducibility of various measures}

Nerve conduction studies serve as a measure of abnormality to document serial changes during the clinical course of polyneuropathy. ${ }^{11}$ Several investigators ${ }^{13-15}$ reported on the reliability of nerve conduction velocity in normal subjects and patients with diabetic polyneuropathy. ${ }^{16-18} \mathrm{~A}$ study of median and peroneal nerves showed good reproducibility of nerve conduction velocity but not of amplitude in patients with diabetic polyneuropathy. ${ }^{16}{ }^{17} \mathrm{~A}$ few studies ${ }^{17-19}$ also yielded an excellent reproducibility of the median and peroneal $\mathrm{F}$ wave latencies in diabetic polyneuropathy. By contrast, amplitude varied considerably for both motor and sensory studies although the use of large electrodes improved the score for compound muscle action potentials. ${ }^{20}$ Of a few reported $\mathrm{F}$ wave studies, all but one ${ }^{18}$ dealt with the experience at a single laboratory, showing variation of up to $10 \mathrm{~m} / \mathrm{s}^{13}$

We conducted a multicentre analysis on intertrial variability of nerve conduction studies in preparation for future drug assessments in diabetic polyneuropathy. ${ }^{21-23}$ All measurements were repeated twice at a time interval of 1-4 weeks by the same examiners, who underwent a hands on

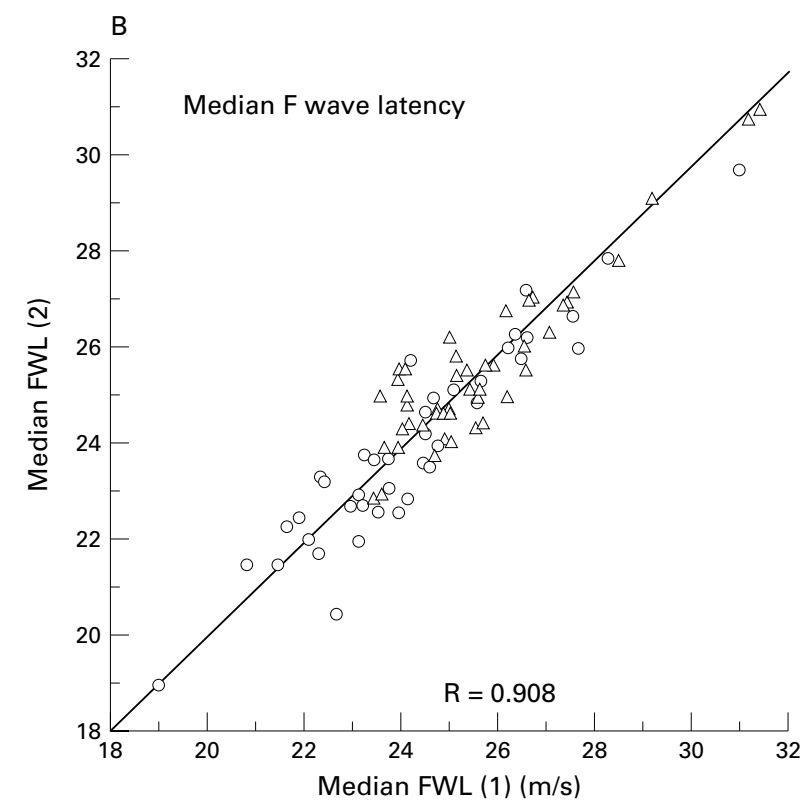

Figure 2 Comparison between the first and second measures of motor nerve conduction velocity (MCV) ( $A$ ) and $F$ wave latency (B). Individual values are plotted showing the first study on the abscissa and second study on the ordinate (from Kimura ${ }^{21}$ courtesy of Kohara et al ${ }^{22}$; data from a multicentre reliability study sponsored by Fujisawa Pharmaceutical). 
workshop to standardise the method. In all, 32 centres participated in the study of 132 healthy subjects ( $63 \mathrm{men})$ and 65 centres in the evaluation of 172 patients with diabetic polyneuropathy (99 men). The protocol consisted of (1) motor nerve conduction studies of the left median and tibial nerves for measurement of amplitude, terminal latency, and minimal $\mathrm{F}$ wave latency, and calculation of motor conduction velocity and $\mathrm{F}$ wave conduction velocity: and (2) antidromic sensory nerve conduction studies of the left median and sural nerves for recording of amplitude and distal latency, and calculation of sensory conduction velocities.

In both the healthy subjects and patients with diabetic neuropathy, amplitude varied most, followed by the terminal latency, and motor and sensory conduction velocites. The minimal $\mathrm{F}$ wave latency showed the least change, with
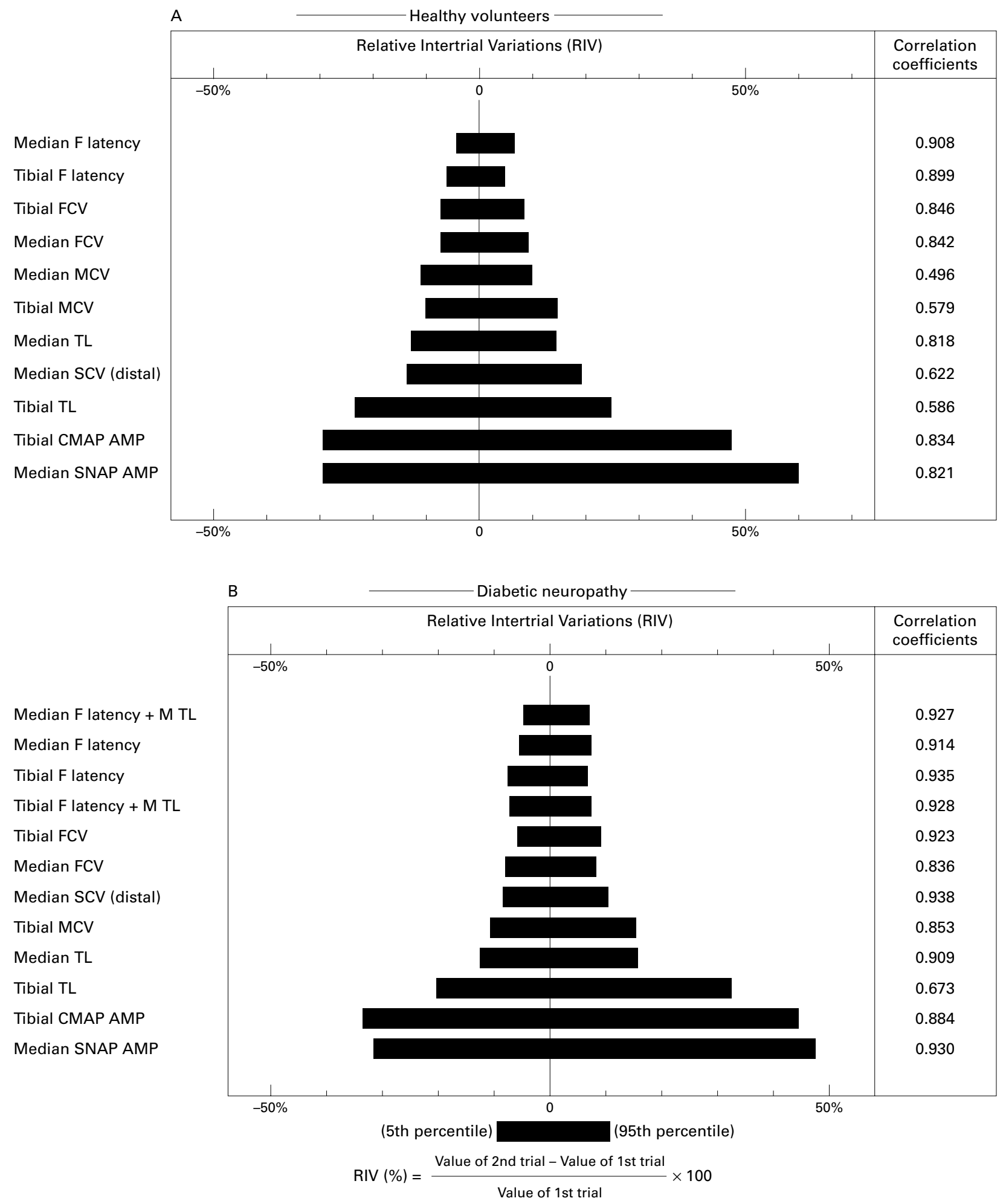

Figure 3 Reproducibility of various measures in healthy volunteers (A) and patients with diabetic neuropathy (B). All studies were repeated twice at a time interval of 1 to 4 weeks to calculate relative intertrial variations and correlation coefficients (from Kimura, ${ }^{21}$ courtesy of Kohara et al ${ }^{22}$; data from a multicentre reliability study sponsored by Fujisawa Pharmaceutical). 
the range of variability of only $10 \%$ for the median nerve and $11 \%$ for the tibial nerve in normal subjects. The corresponding values were $12 \%$ and $14 \%$, respectively, in patients with diabetic polyneuropathy. These results support the hypothesis that the minimal $\mathrm{F}$ wave latency serves as the most reliable measure of nerve conduction for a sequential study in the same subjects. When evaluating indivdual patients against a normal range established in a group of subjects, however, $\mathrm{F}$ wave conduction velocity suits better, because it minimises the effect of limb length. Alternatively, some prefer the use of a nomogram plotting the latency against the height as a simple, albeit indirect, measure of limb length.

\section{Relative intertrial variations $v$ intraclass correlation coefficiency}

Of the two independent indices used in the assessment of reproducibility, relative intertrial variation (RIV) is calculated as a variation of measurements expressed in percentages of the difference between V1 and V2 over the mean value of the two. Thus:

$$
\mathrm{RIV}(\%)=100(\mathrm{~V} 2-\mathrm{V} 1) / 0.5(\mathrm{~V} 1+\mathrm{V} 2)
$$

where V1 and V2 represent the values of the first and the second measurements of the pair. The ranges of RIV within $\pm 10 \%$ usually indicate a higher precision.

The variance of a given procedure consists of two components: among subject variance $\left(\sigma_{\mathrm{s}}^{2}\right)$ and intertrial error $\left(\sigma_{\varepsilon}^{2}\right)$. Measures having larger interindividual differences are expected to show a greater intertrial variability. The calculation of the other index, intraclass correlation coefficiency (ICC), takes this into consideration as follows to partially offset the effects of a large variability among different subjects. Thus:

$$
\text { ICC }=\sigma_{\mathrm{s}}{ }^{2} /\left(\sigma_{\mathrm{s}}^{2}+\sigma_{\varepsilon}^{2}\right)
$$

The value exceeding 0.9 indicates a reliable measure although, as seen from the equation, this may reflect a large among subject variance rather than a small intertrial error.

Figure 2 illustrates some examples of the individual data comparing the reproducibility of motor nerve conduction velocities and $F$ wave latencies measured on two separate occasions. Figure 3 summarises the 5 th to 95 th percentiles of RIV and ICC in both healthy subjects and patients with diabetic neuropathy. The measures showing the range of RIV within $\pm 10 \%$ included $F$ wave latency and $F$ wave conduction velocity of both median and tibial nerves and sensory conduction velocity of the median nerve. In general, amplitudes showed a greater variation than latencies or nerve conduction velocities. Similarly, ICC exceeded 0.9 for $\mathrm{F}$ wave latency of the median and tibial nerves in both the healthy subjects and the patients. In some measures, a large among subject variance of the amplitudes made $\sigma_{\mathrm{s}}^{2}$ much greater than $\sigma_{\varepsilon}^{2}$, leading to a high ICC despite a considerable intertrial variability. These included the amplitude of the median nerve sensory action potential and median and tibial nerve compound muscle action potentials.

Based on these findings, we conclude that a high ICC, indicating a statistical correlation between two measurements, ${ }^{17}$ does not necessarily imply a good reproducibility. The calculation of RIV in addition to ICC helps to detect the indices with an acceptable degree of reproducibility on both accounts. Thus, a sequential study must exclude any measurements with a wide RIV regardless of ICC values. $F$ wave latencies of the median and tibial nerves are the most reliable measures showing a small RIV $( \pm 10 \%)$ and a large ICC $(>0.9)$.

\section{Clinical consideration}

Our data indicate that the length of the nerve segment under study dictates the accuracy and sensitivity of measurement. Although studies of shorter or longer segment pose technical merits and demerits, the choice seems to depend entirely on the pattern of the conduction abnormalities. Short distances magnify focal conduction abnormalities despite increased measurement error, and long distances, although insensitive to focal lesions, provide better yields and reliability for a diffuse or multisegmental process. These findings also underscore the importance of choosing nerve stimulation techniques best suited for detecting the clinically supsected lesion. Thus, electrophysiological studies serve well only when conducted as an extension of the history and physical examination, which provide an overall orientation for subsequent physiological evaluation.

1203 Park City Uji Byoudoin, 169 Uji Myouraku Uji Shi, Kyoto 611-0021 Japan

1 Lambert EH. Diagnostic value of electrical stimulation of motor nerves. Electroencephalogr Clin Neurophysiol 1962;(suppl 22):9-16.

2 Daube JR. Nerve conduction studies. New York:Churchill Livingstone, 1980 . 229-64.

3 Kimura J. The carpal tunnel syndrome: localization of conduction abnormalities within the distal segment of the median nerve. Brain 1979;102:619-35.

4 Imaoka H, Yorifuji S, Takahashi M, et al. Improved inching method for the diagnosis and prognosis of carpal tunnel syndrome. Muscle Nerve 1992;15: $318-24$.

5 Ross MA, Kimura J. AAEM case report 2. The carpal tunnel syndrome. Muscle Nerve 1995;18:567-73.

6 Seror P. Orthodromic inching test in mild carpal tunnel syndrome. Muscle Nerve 1998;21:1206-8.

7 Campbell WW, Pridgeon RM, Sahni KS. Short segment incremental studies in the evaluation of ulnar neuropathy at the elbow. Muscle Nerve 1992; 15:1050-4.

8 Kanakamedala RV, Simons DG, Porter RW, et al. Ulnar nerve entrapment at the elbow localized by short segment stimulation. Arch Phys Med Rehabil the elbow localize

9 Kanakamedala RV, Hong C-Z. Peroneal nerve entrapment at the knee localized by short segment stimulation. Am f Phys Med Rehabil 1989;68:116-22.

10 Kaji R, Oka N, Tsuji S, et al. Pathological findings at the site of conduction block in multifocal motor neuropathy. Ann Neurol 1993;33:152-8.

11 Kimura J, Yamada T, Stevland NP. Distal slowing of motor nerve conduction velocity in diabetic polyneuropathy. F Neurol Sci 1979;42:291302

12 Fraser JL, Olney, RK. The relative diagnostic sensitivity of different F-wave parameters in various polyneuropathies. Muscle Nerve 1992;15:912-18.

13 Bleasel A, Tuck R. Variability of repeated nerve conduction studies. Electroencephalogr Clin Neurophysiol 1991;81:417-20.

14 Bril V, Ellison R, Ngo M, et al. Electrophysiological monitoring in clinical trials. Muscle Nerve 1998;21:1368-73.

15 Salerno DF, Werner RA, Albers JW, et al. Reliability of nerve conduction studies among active workers. Muscle Nerve 1999;22:1372-9.

16 Claus D, Mustafa C, Vogel W, et al. Assessment of diabetic neuropathy definition of norm and discrimination of abnormal nerve function. Muscle Nerve 1993:16:757-68.

17 Dyck PJ, Kratz KM, Lehman KA, et al. The Rochester diabetic neuropathy study: design, criteria for types of neuropathy, selection bias, and reproducibility of neuropathic tests. Neurology 1991;41:799-807.

18 Valensi P, Attali J-R, Gagant S. Reproducibility of parameters for assessment of diabetic neuropathy. Diabet Med 1993;10:933-9.

19 Andersen H, Stalberg E, Falck B. F-wave latency, the most sensitive nerve conduction parameter in patients with diabetes mellitus. Muscle Nerve 1997;20:1296-302.

20 Tjon-A-Tsien AML, Lemkes HHPJ, van der Kamp-Huyts AJC, et al. Large electrodes improve nerve conduction repeatability in controls as well as in patients with diabetic neuropathy. Muscle Nerve 1996;19:689-95.

21 Kimura J. Facts, fallacies, and fancies of nerve conduction studies: $21 \mathrm{st}$ annual Edward H Lambert lecture. Muscle Nerve 1997;20:777-87.

22 Kohara N, Kimura J, Kaji R, et al. Multicenter analysis on intertrial variability of nerve conduction studies: healthy subjects and patients with diabetic polyneuropathy. In: Kimura J, Shibasaki H, eds. Recent advances in clinical polyneuropathy. In: Kimura J, Shibasaki H, eds. Recent advances

23 Kohara N, Kimura J, Kaji R, et al. F-latency serves as the reproducible measure in nerve conduction studies of diabetic polyneuropathy: multicenter analysis in healthy subjects and patients with diabetic polyneuropathy. Diabetologia 2000;43:915-21.

24 Winer BJ. Statistical principles in experimental design. 2nd ed. New York: McGraw-Hill, 1972:283-9.

25 Kimura J. Kugelberg lecture: principles and pitfalls of nerve conduction studies. Electroencephalogr Clin Neurophysiol 1998;106:470-6. 